\title{
Hints and challenges in heavy flavor physics
}

\section{Shoji Hashimoto*}

High Energy Accelerator Research Organization (KEK), Tsukuba 305-0801, Japan SOKENDAI (Graduate University for Advanced Studies), Tsukuba 305-0801, Japan

E-mail: shoji.hashimoto@kek.jp

Heavy flavor physics entered a new era when the Belle II experiment observed its first collision. There are several hints found so far by BaBar, Belle, and LHCb in particular, that suggest the physics beyond the Standard Model appearing in the loop processes at short distances. They will be further tested by higher precision experiments in the coming years, while the role of lattice QCD is to understand the long-distance physics quantitatively so that one can unambiguously isolate the short-distance physics from the experimental data. I summarize the status towards this goal and then look at the challenges we are facing.

The 36th Annual International Symposium on Lattice Field Theory - LATTICE2018

22-28 July, 2018

Michigan State University, East Lansing, Michigan, USA.

${ }^{*}$ Speaker. 


\section{Introduction}

On April 26, 2018, the Belle II detector observed the first collision of an electron-positron pair accelerated by the SuperKEKB accelerator. This is the event that opens a new era of heavy flavor physics. The SuperKEKB is designed to deliver 50 times more luminosity than the previous KEKB accelerator did. At the time of the conference, the Phase 2 operation of SuperKEKB, which is the run with Belle II but without its inner-most vertex detectors, had just been finished and the machine is scheduled to restart in March 2019 with fully equipped Belle II. The plan then is to accumulate $50 \mathrm{ab}^{-1}$ within 5-6 years of running.

In $B$ meson decays, there are already several hints of new physics. A well-known example is the $B \rightarrow D^{(*)} \tau \nu$ decay branching fractions, which show an enhancement compared to the Standard Model expectation [1,2]. Because of $\tau$ lepton mass, the form factor uncertainty does not completely cancel even in the ratio between the branching fractions for $B \rightarrow D^{(*)} \tau \nu$ and $B \rightarrow D^{(*)} \mu \nu$, and the lattice calculations by Fermilab/MILC [3] and HPQCD [4] have been used to predict the Standard Model value. The experimental data show a tension of about $3.8 \sigma$.

For the rare decays $B \rightarrow K^{(*)} \ell^{+} \ell^{-}$, some hints of new physics have been found in the lepton flavor universality test, $R\left(K^{(*)}\right)=\Gamma\left(\bar{B} \rightarrow \bar{K}^{(*)} \mu^{+} \mu^{-}\right) / \Gamma\left(\bar{B} \rightarrow \bar{K}^{(*)} e^{+} e^{-}\right.$) (the most precise LHCb observations to date are $[5,6]$; see also the references therein) as well as an angular asymmetry $P_{5}^{\prime}$ of $B \rightarrow K^{*} \mu^{+} \mu^{-}$(results from LHCb [7] and Belle [8], CMS [9] and ATLAS [10]).

A more relevant quantity to the lattice calculation is the branching fraction of the $B \rightarrow K \ell^{+} \ell^{-}$ decay mode. The differential decay rate is estimated using a lattice calculation and other constraints. The experimental data by CDF, Belle, BaBar and LHCb look consistently lower as shown in Figure 6 of [11]. This comparison is made away from the region of charmonium resonances $\left(J / \psi\right.$ and $\left.\psi^{\prime}\right)$, since there are huge contributions from $B \rightarrow \psi^{\left({ }^{\prime}\right)} K \rightarrow \ell^{+} \ell^{-} K$ due to a Cabbibo allowed process $b \rightarrow c \bar{c} s$. Quantitative estimate of such long-distance contribution is still missing, and a separation of short-distance physics (new physics) and long-distance physics (QCD) remains a difficult problem.

The challenges for lattice QCD in the context of heavy flavor physics are twofold. One is the precision frontier. For the simple quantities like decay constants and semileptonic form factors, one wants to achieve the precision as good as that the corresponding experiments provide. The other is the study to understand unknowns, which includes the long-distance effect as well as the puzzles between the inclusive and exclusive determination of $\left|V_{c b}\right|$ and $\left|V_{u b}\right|$. For such quantities, the way to proceed might not be unique, and even a crude calculation could be helpful.

In this talk I describe my (sketchy) understanding of the status of these fronts. Section 2 summarizes the status of the precision frontier. I take the most recent calculation of the $B$ meson decay constant as an example in order to consider about what has been and what will be the main challenges. I then move to the discussion of the exclusive determination of $\left|V_{c b}\right|$ in Section 3. I focus on this quantity because there was a significant progress and associated confusions in the last couple of years. Section 4 is devoted to more complicated quantities, such as the inclusive $B$ meson decay calculation and the long-distance effect in $B \rightarrow K \ell^{+} \ell^{-}$.

I have to mention that this is not a comprehensive review. I am not going to provide averages of lattice calculations. For the averages, the Flavour Lattice Averaging Group (FLAG) reviews most 
recent calculations and provide up-to-date averages every 2-3 years. The last edition (FLAG3) was published in 2016 [12] and the next edition (FLAG4) is scheduled to appear in early 2019.

\section{Precision frontier}

The biggest news in the precision frontier in the last couple years came from the Fermilab/MILC collaboration, which reported a calculation of the $B$ meson decay constant at the level of precision better than $1 \%$ for the first time [13]. The result is $f_{B}=189.4 \pm 1.4 \mathrm{MeV}$, which shows a significant improvement compared to the previous average by FLAG [12], $192.0 \pm 4.3 \mathrm{MeV}$ for $N_{f}=2+1$ or $186 \pm 4 \mathrm{MeV}$ for $N_{f}=2+1+1$ (based on the calculations of Fermilab/MILC [14], $\mathrm{RBC}[15,16]$, and HPQCD [17, 18]).

\subsection{Discretization effect for heavy}

Achieving such precision is highly non-trivial, because the heavy quarks are much harder to treat on the lattice due to their short Compton wavelength. This is indeed the reason of long history of works to develop and test effective theories for heavy quarks on the lattice. The solution adopted in this work, on the other hand, is to use the Highly Improved Staggered Quark (HISQ) action [19] for both heavy and light quarks, and to extend the simulations on the lattices with small lattice spacings down to $a=0.042 \mathrm{fm}$ at the physical pion mass. The computational effort spent for this work, 70 TFlops $\times$ yr for the ensemble generation plus another 70 TFlops $\times$ yr for the measurements (both in the unit of sustained TFlops), was of course a key for the success, but does not explain everything because the highest lattice cutoff is still around $5 \mathrm{GeV}$ and not enough to satisfy the condition $a m_{b} \ll 1$ for the physical $b$ quark mass $m_{b}$.

The discretization effect for heavy quark may be estimated according to the so-called Fermilab interpretation [20], that applies the Heavy Quark Effective Theory (HQET) for the lattice regularization with finite $a m_{b}$. The leading discretization effect for heavy HISQ quarks is then estimated to be of $O\left(\alpha_{s} a^{2} m^{2}\right)$. Numerically, it is not small for the physical $b$ quark mass. Even for a fictitious " $b$ " quark that has an intermediate mass of $3 \mathrm{GeV}$, it gives a rough estimate of about $7 \%$. (Other parameters are taken $\alpha_{s} \sim 0.2$ and $a^{-1} \simeq 5 \mathrm{GeV}$ corresponding to the Fermilab/MILC calculation.)

In order to achieve the sub- $\%$ precision, therefore, the discretization error has to be eliminated by a continuum extrapolation. In the calculation of the Fermilab/MILC collaboration, this is done with a number of lattice data calculated at various " $b$ " quark masses between the charm quark mass and the physical $b$ quark mass. The lattice spacing covers a wide range from $0.15 \mathrm{fm}$ down to $0.03 \mathrm{fm}$, which enable them to fit the data globally allowing the terms of $1 / m^{k}(k \geq 0)$ and $\alpha_{s}(m a)^{n}$ $(n \geq 2)$ to account for the physical $1 / m$ dependence as well as the discretization effects. The heavy quark mass one can reach at each lattice spacing without too large discretization effect and can be used in the analysis is limited by a condition that $m a$ is below some value. The Fermilab/MILC collaboration chose $m a<0.9$.

Fig. 1 demonstrates this global fit. One can see that the results at growing lattice cutoffs tend to follow a single curve more closely, and they finally show the envelope to represent the continuum limit. The cyan curve is their estimate of the continuum limit obtained by the global fit. A surprising observation is that the deviation from this continuum limit is much smaller than what we estimate using the effective theory. For instance, for our fictitious $b$ quark at " $m_{b} "=3 \mathrm{GeV}$, 


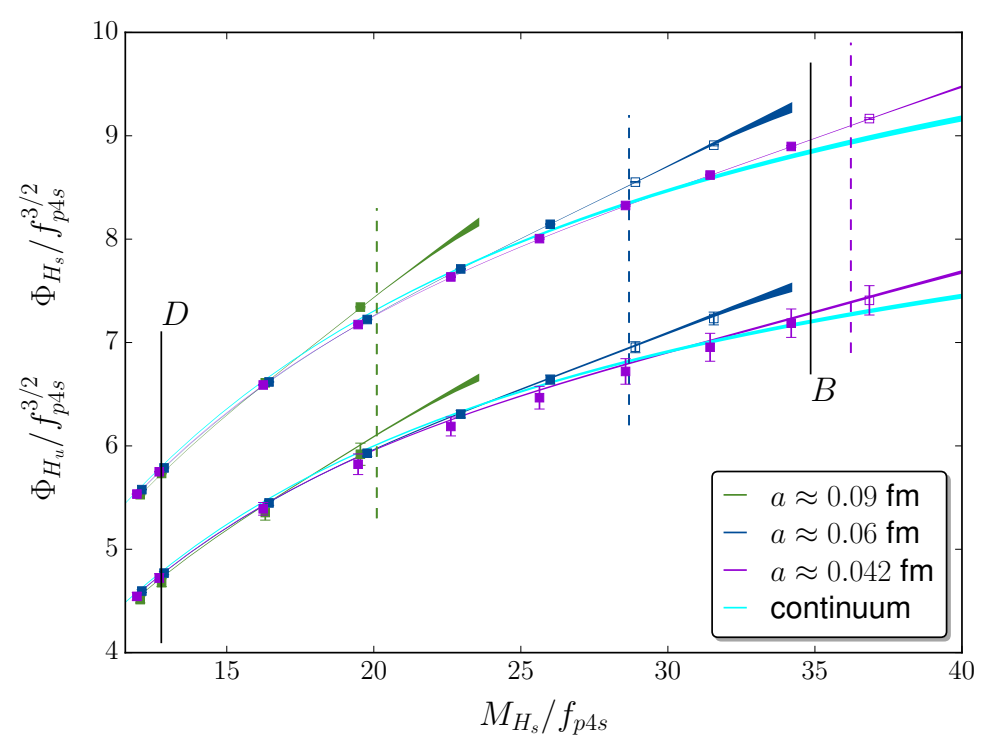

Figure 1: Heavy quark and continuum extrapolation of heavy meson decay constants $\Phi_{H} \equiv f_{H} \sqrt{m_{H}}$. The plot is from Fermilab/MILC [13]. Two bunches of curves represent those for $B_{s}$ (above) and $B_{u}$ (below). For each case, the results at various heavy quark masses obtained at three different lattice spacings are plotted. The continuum limit obtained by a global fit is shown by cyan curves.

the meson mass is roughly in the middle between the physics $M_{D}$ and $M_{B}$. The deviation from the continuum limit (cyan) seems to be much smaller than the estimated 7\% at $a \simeq 0.042 \mathrm{fm}$ (blue) and is almost invisible in the scale of Fig. 1. (The size of the discretization effect is actually about $3 \%$ or less even including the coarser lattices of $a \simeq 0.09 \mathrm{fm}$.)

This small discretization effect may be partly due to the fact that the renormalization constant of the axial-vector current is automatically determined when one uses the same relativistic quark action for both heavy and light. The bulk of the discretization effect of $O\left(\alpha_{s} a^{2} m^{2}\right)$ may be absorbed in this method. This might be a lucky situation that happens only for the decay constant and might not be the case for other quantities.

I also mention that this strategy to employ the HISQ heavy quark even for "bottom" and to extrapolate to the physical point was originated from the pioneering work by the HPQCD collaboration [21]. The Fermilab/MILC collaboration extended this program to smaller lattice spacings and to higher statistics, and finally achieved the precision calculation.

There are various strategies for the treatment of heavy quarks on the lattice. For the effective theory approaches, such as the NRQCD action [22], the Fermilab action [20] and its variants, one has to match the action and operators to reproduce the relativistic continuum fermion. The matching is usually carried out using perturbation theory, and the error of $O\left(\alpha_{s}^{2}\right)$ remains when the one-loop corrections are included. (Note also that this can be improved by the so-called mostly non-perturbative matching, which means that the currents made of degenerate quarks are nonperturbatively renormalized and possible deviations for non-degenerate quarks are estimated by perturbation theory.) The method to perform the matching completely non-perturbatively has been formulated by the ALPHA collaboration [23, 24, 25, 26]. It requires dedicated simulations with various volumes and lattice spacings, and takes time to carry out. Finally, the relativistic approach 

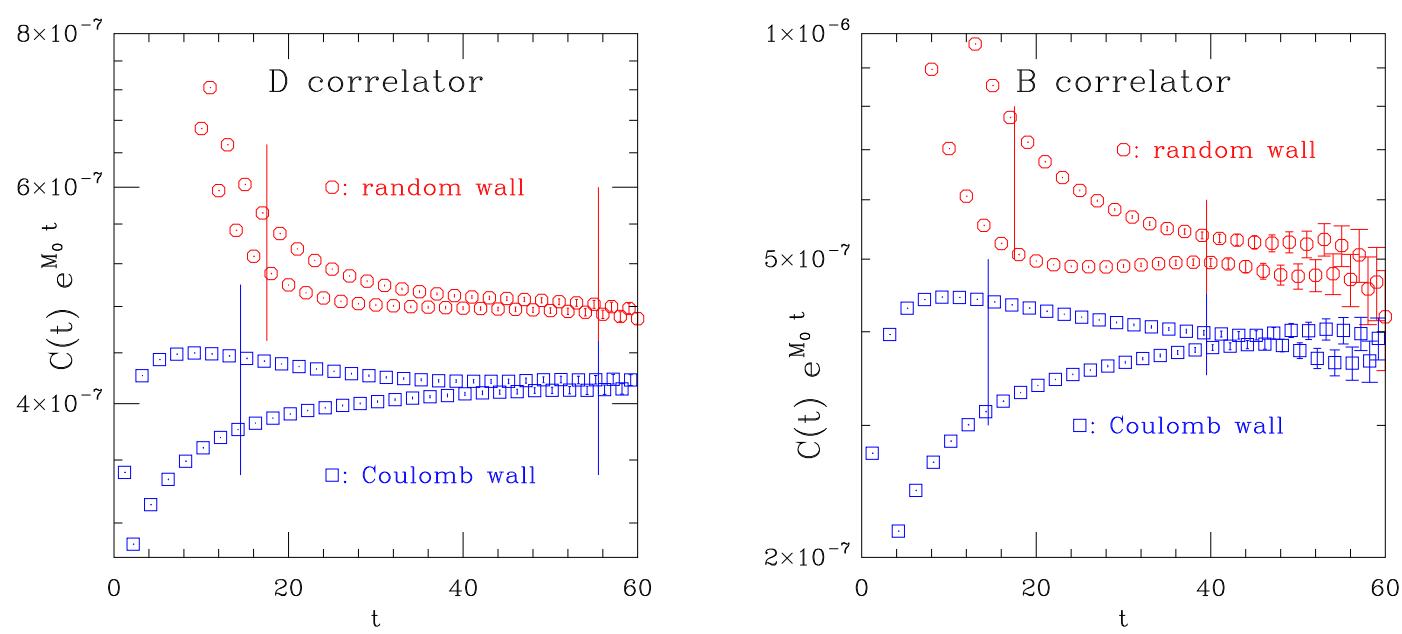

Figure 2: $D$ meson (left) and $B$ meson (right) correlator divided by their ground-state exponential function $e^{-M_{0} t}$. The plots are from Fermilab/MILC [13]. They are obtained on a fine $(a \simeq 0.042 \mathrm{fm})$ lattice and at physical pion mass. Circles and squares are with different sources, and the zigzag structure is due to the staggered fermion.

has become more common as the computational power increases. While one needs small lattice spacings in order to suppress discretization effects, the non-perturbative matching of the operators is much simpler than the effective theory approaches. Such a brute-force approach could be a simple solution in some cases, and the most recent Fermilab/MILC calculation of $f_{B}$ is a prominent example.

\subsection{Growing noise and excited-state contamination}

The statistical error poses more problems for heavy quarks. According to Lepage's analysis [27], which was numerically confirmed in [28], one can predict how rapidly the statistical noise grows in the correlation functions. For the heavy-light meson correlator $C_{H L}(t)$, the statistical noise $\delta C_{H L}(t)$ grows as

$$
\frac{\delta C_{H L}(t)}{C_{H L}(t)} \propto \exp \left[\left(m_{B}-\frac{m_{\eta_{b}}+m_{\pi}}{2}\right) t\right] .
$$

The noise "doubling time"1 $m_{B}-\left(m_{\eta_{b}}+m_{\pi}\right) / 2$ is determined by the physical mass spectrum and there is nothing to do with the lattice details. Numerically, it is about $0.4 \mathrm{fm}$ for the $B$ meson, which is much worse compared to the $D$ meson $(0.65 \mathrm{fm})$. The growing noise, especially for the $B$ meson, can be clearly seen in the data by Fermilab/MILC [13], which is reproduced in Figure 2.

As a result, one is forced to fit the correlator before the ground state dominates, in order not to lose the signal at all. Such a fit including the excited-state contamination modeled by multiple exponential functions is a numerically difficult problem. In the $f_{B}$ calculation by Fermilab/MILC [13], the multi-exponential fit is applied with five distinct states included. The fit range shown by vertical lines in the plots; the maximal time separation is about $1.6 \mathrm{fm}$ for the $B$ meson (right) where the plateau is not yet reached. Two of the states included are opposite-parity states due to

\footnotetext{
${ }^{1}$ Actually the time that the noise becomes $\times 2.7$.
} 
the use of the staggered fermion, and the other two are the excited states of the target $B$ meson. Assuming the physical mass spectrum, the candidates for these low-lying excited states are the two-body $B^{*} \pi$ states with finite relative momenta between $B^{*}$ and $\pi$. On a large lattice of size $L \simeq$ $6 \mathrm{fm}$, as used by the Fermilab/MILC calculation, they start from $300 \mathrm{MeV}$ above the ground state and their spectrum is nearly continuum like, i.e. a dense distribution of different energy eigenstates are expected. The excited-state energy obtained by the fit is consistent with this band of states, but they are not separately identified. The extraction of the ground state would be stable even though the excited states are not precisely accounted for, but the crucial question is whether the statistical error thus estimated is valid especially when the plateau is not cleanly identifiable. Such careful study would become more relevant for many quantities as the numerical precision improves.

The lattice simulation at the physical pion mass is a big challenge for us if not a nightmare. First of all, with the small pion mass the signal-to-noise ratio becomes worse for the reason just described, and one has to fit the lattice correlators before plateau is reached. This itself is fine, but the situation gets even worse because the excited-state energy is made lower as $m_{\pi}$ and the isolation of the the ground state needs larger time separations. These excited states become dense when the lattice volume is large as required to keep the finite volume effect under control. It means that the multi-exponential function is no longer a valid description of the correlator.

This problem had already become manifest for baryons. The analysis of the noise growth rate implies that the "doubling time" is about $0.27 \mathrm{fm}$ for nucleon, even smaller than for the $B$ meson. In a recent computation of nucleon correlator at the physical point, i.e. the one by the PNDME collaboration [29], no clear plateau is visible before signal dies at around the time separation of $1.5 \mathrm{fm}$. The authors attempted multi-exponential fits, and the resulting excited-state energy was not consistent with what one expects from the $N \pi$ continuum states. An interpretation might be that the interpolating operator used in this work has small overlap with such two-body states, and even higher-energy states show up in the data. But, then, one should ask if there is any bias due to the unseen continuum-like states. Certainly, a better understanding of the correlator at short distances is desired.

Another important warning may be found in the computation of the axial charge of nucleon. Bär gave a plenary talk at Lattice 2017 [30] concerning a possible bias due to the correlator fit at too short source-sink separations (see also [31,32] and a talk at this conference [33]). Within chiral perturbation theory (ChPT) one can compute the contribution of the $N \pi$ states to the correlator relevant to the calculation of the nucleon axial charge $g_{A}$. With finite source-sink separations, but larger than $2 \mathrm{fm}$, he estimated a bias that overestimates $g_{A}$ by about 2-7\%. Many of the lattice computations, on the other hand, gave results lower than the experimental value by about 3-10\%. A potentially serious problem here is that the source-sink separation adopted in these calculation is too small, $\lesssim 1.5 \mathrm{fm}$, to safely apply ChPT. (The separation between the source (or sink) and the current is less than half of this.) Since there is no a priori criterion for the necessary time separation, the nucleon $g_{A}$ would provide an important benchmark problem for lattice computation, for which the consistency with a theoretical estimate has not been established.

A similar, or even tougher, problem is the calculation of heavy baryon decay form factors, such as those of $\Lambda_{b} \rightarrow p \ell v$. The noise doubling time for $\Lambda_{b}$ is about $0.25 \mathrm{fm}$, which is even shorter than that for nucleon. Because of such a bad signal-to-noise, the authors of [34] had to fit the correlators at the source-sink separations between 0.5 and $1 \mathrm{fm}$, much smaller than the separation typically 
adopted in the $B \rightarrow \pi \ell v$ form factor calculations. They numerically confirmed the stability of the ground-state signal within the existing data, but more stringent consistency checks including those of excited-state energy would be highly desired since the fit range does not even touch the plateau region.

\subsection{Heavy-to-light decays}

Semileptonic decays of heavy mesons to light mesons, such as $B \rightarrow \pi \ell v$ and $B_{s} \rightarrow K \ell v$, are challenging quantities for the lattice calculation. It is not only due to the heavy initial $B$ meson but the final state ( $\pi$ or $K$ for these examples) can have relatively large momenta. With a finite momentum, the noise growth rate of pion correlator, for instance, is given as $\delta C_{\pi}(t, \mathbf{p}) / C_{\pi}(t, \mathbf{p}) \propto$ $\exp \left[\left(E_{\pi}(\mathbf{p})-m_{\pi}\right) t\right]$, and it sets the limit on the value of the momentum transfer $q^{2}$ one can reach. The maximum momentum of the final state pion is typically around $600-800 \mathrm{MeV} / c$, which is much lower than the kinematically allowed maximum $\sim 2.6 \mathrm{GeV} / c$. Previous calculations, such as those by RBC/UKQCD [35], Fermilab/MILC [36], ALPHA [37], actually observed the growing noise for larger momenta. These groups therefore restricted themselves for small recoil momenta (and thus large $q^{2}$ ) and fit the plateau. The possibility of fitting including much shorter timeseparations even without finding plateaus has not been fully explored.

At this conference, there have been presentations on the heavy-to-light form factor calculations by Fermilab/MILC (Gelzer et al. [38], see also [39, 40] for preliminary results at Lattice 2017), RBC/UKQCD (Witzel et al. [41]), JLQCD (Colquhoun et al. [42], see also [43]), HPQCD (Bouchard et al. [44], see also [45] for a full paper), as well as ETM (Riggio et al. [46], see also [47] for a full paper).

To conclude this section, I emphasize that precise calculation has become realistic even for $b$ quark. An important factor for this development is the use of the HISQ action combined with a large amount of computer resources. Results with other lattice formulations are highly desired to cross-check the results. Extension of the calculation to more complicated problems, such as the heavy-to-light form factors, is more challenging mainly due to the noise problem. The "fitbefore-plateau" strategy will be used more commonly for these quantities, and we need thorough understanding of the excited-state contamination especially those from the continuum, such as $B^{(*)} \pi$, states.

\section{3. $\mathbf{A}\left|V_{c b}\right|$ story}

One of the key CKM elements, $\left|V_{c b}\right|$, can either be determined using the exclusive decay processes $B \rightarrow D^{(*)} \ell v$ or the inclusive decay rate of $B \rightarrow X_{c} \ell v$. The exclusive determination relies on the constraints given by heavy quark symmetry and lattice calculations.

In the limit of infinitely heavy quarks, $m_{b}, m_{c} \rightarrow \infty$, the heavy-to-heavy meson transition form factors, for both $B \rightarrow D \ell v$ and $B \rightarrow D^{*} \ell v$, can be written in terms of an universal function $\xi(w)$, called the Isgur-Wise function [48, 49]. This is a remarkable result of the heavy quark symmetry. Here, the argument $w$ is an inner product of initial and final meson velocities, $w \equiv v \cdot v^{\prime}$. In the zero-recoil limit, $w=1$, the process is nothing but an insertion of a temporal vector current between static mesons, and the form factor is normalized to one due to vector current conservation: $\xi(1)=1$. The correction to the $m_{b, c} \rightarrow \infty$ limit starts from $O\left(1 / m^{2}\right)$, i.e. no $O(1 / m)$ corrections 
[50], in the zero-recoil limit, so that the size of the correction is suppressed by a factor of $\bar{\Lambda}^{2} / m_{c}^{2}$ with $\bar{\Lambda}$ a typical QCD scale and thus expected to be about $10 \%$ or less. The standard strategy for a precise determination of $\left|V_{c b}\right|$ is therefore to measure $\left|V_{c b}\right| \mathscr{F}(w)$ in the experiments $(\mathscr{F}(w)$ stands for the form factor at finite $m_{b, c}$ ). One extrapolates the data to the $w \rightarrow 1$ limit and inputs an theoretical estimate for $\mathscr{F}(1)$ to extract $\left|V_{c b}\right|$. In this way one can avoid hadronic uncertainty as much as possible.

The best theoretical estimate for $\mathscr{F}(1)$ now comes from lattice calculation, and the lattice calculation itself gets benefits from the heavy quark symmetry. For instance, a double ratio of the zero-recoil form factors

$$
\left|h_{+}(1)\right|^{2}=\frac{\left\langle D\left|\bar{c} \gamma_{0} b\right| \bar{B}\right\rangle\left\langle\bar{B}\left|\bar{b} \gamma_{0} c\right| D\right\rangle}{\left\langle D\left|\bar{c} \gamma_{0} c\right| D\right\rangle\left\langle\bar{B}\left|\bar{b} \gamma_{0} b\right| \bar{B}\right\rangle}
$$

becomes unity in the heavy quark limit. The left-hand side doesn't have a denominator because the current conservation of the flavor-conserving vector current normalizes the corresponding form factor to 1 . Away from the heavy quark limit, a symmetry under the exchange between $b$ and $c$ forbids the correction of $O(1 / m)$. By designing the lattice calculation such that this symmetry is manifest, one essentially calculates the deviation from the heavy quark limit, $\left|h_{+}(1)\right|^{2}-1$, which is of $O\left(1 / m_{b, c}^{2}\right)$ [51, 52].

Unquenched lattice results for the zero-recoil form factor have so far been obtained by Fermilab/MILC (for $B \rightarrow D \ell v$ [3] and for $B \rightarrow D^{*} \ell v$ [53]) and HPQCD (for $B \rightarrow D \ell v$ [4] and for $B \rightarrow D^{*} \ell v$ [54]). Combined with the experimental averages provided by the HFLAV group [1], the results for $\left|V_{c b}\right|$ are $0.0398(10)(14)$ from $B \rightarrow D \ell v$ and 0.0391(5)(5) from $B \rightarrow D^{*} \ell v$. It is to be compared with another determination, 0.0422(8), from the inclusive $B$ meson decays [1]. If we take them at their face values, there is a tension of about $3 \sigma$ between the exclusive and inclusive determinations. This is a long-standing puzzle in the determination of $\left|V_{c b}\right|$ that prevents us from performing more precise tests of the CKM unitarity.

More recently, the situation was changed by a new analysis of $B \rightarrow D^{*} \ell v$ by Belle [55]. They created an unfolded data of the differential decay rate available in the form that theorists can attempt their own analysis. Several theorists have actually studied the fit of the experimental data of $\left|V_{c b}\right|^{2} \mathscr{F}^{2}(w)$ using various ansatz for the functional form of $\mathscr{F}(w)$, and found that the results depend on the details of the fit function $[56,57,58]$.

The commonly used fit ansatz in the experimental analyses had been that of Caprini, Lellouch, Neubert (CLN) [59], which was developed from a more general formulation by Boyd, Grinstein, Lebed (BGL) [60]. The both use the idea of the dispersive bound [61, 62], which relates twopoint functions of the form $\left\langle J^{\bar{c} b} J^{\bar{b} c}\right\rangle$ to a sum of the matrix elements $\left|\left\langle 0\left|J^{\bar{c} b}\right| \bar{B}_{i}^{(*)} D_{j}^{(*)}\right\rangle\right|^{2}$. Here, the subscripts $i$ and $j$ denote possible states allowed by symmetries. Since the matrix elements are written in terms of the corresponding form factors, this relation gives certain constraints on the form factors integrated over their kinematical variables. The constraints are used such that the functional form of $\mathscr{F}(w)$, for instance, is well approximated by a polynomial of a variable $z=(\sqrt{1+w}-\sqrt{2}) /(\sqrt{1+w}+\sqrt{2})$ truncated at some order. On top of that, the CLN ansatz incorporates some estimates of the $1 / m$ corrections from heavy quark effective theory, and thus puts tighter constraints than the BGL does. For instance, the form of $h_{A_{1}}(w)$, a dominant part of $\mathscr{F}(w)$, is parametrized by only two parameters up to $O\left(z^{3}\right)$, i.e. the slope of $\mathscr{F}(w)$ at $w=1$ determines its curvature too. 


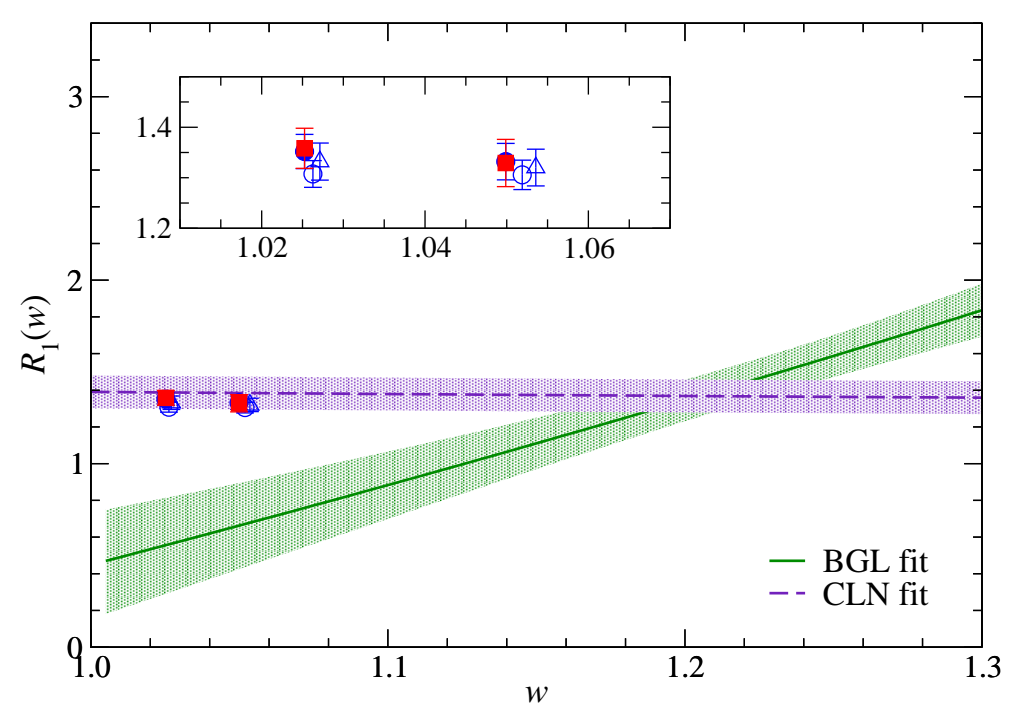

Figure 3: $R_{1}(w) \equiv h_{V}(w) / h_{A_{1}}(w)$ extracted from the BGL and CLN fits [58] of the Belle data (bands). The lattice results by Kaneko et al. [63] are shown by the points (and also magnified in the inset). A plot from [63].

The new observation by $[56,57,58]$ is that the BGL fit yields a higher value of $\left|V_{c b}\right|$, which is consistent with the inclusive determination. Since the BGL ansatz is more general, and thus more model-independent, one might argue that this is the solution of the $\left|V_{c b}\right|$ puzzle. The situation is, however, not that simple. The same BGL fit also gives results on the parameters for which an estimate from HQET at $O(1 / \mathrm{m})$ is available, and the results indicate unnaturally large $O(1 / \mathrm{m})$ corrections. An example is shown in Figure 3, where $R_{1}(w) \equiv h_{V}(w) / h_{A_{1}}(w)$ is plotted. $\left(h_{V}(w)\right.$ and $h_{A_{1}}(w)$ are form factors to represent some kinematical structures of $B \rightarrow D^{*} \ell v$.) This ratio becomes unity in the heavy quark limit, and the leading correction is expected to be of $O\left(\bar{\Lambda} / m_{c}\right)$, while the plot shows that the BGL fit of the Belle data [58] leads to an $O(1)$ deviation from 1.

More extensive test of the heavy quark symmetry relations among form factors is necessary on both experiment and lattice, which was also emphasized by [64]. One example is $R_{1}(w)$ shown in Figure 3, where the results of lattice calculation by Kaneko et al. [63] are plotted together with the BGL and CLN fits of [58]. It is clear that the lattice results prefer the CLN fit (See also [64]), and the latest BGL fits that yield the higher value of $\left|V_{c b}\right|$ are not totally satisfactory.

To summarize, the situation of the $\left|V_{c b}\right|$ determination is yet unclear. The lattice calculation is the main theoretical source of information to resolve the puzzle. In order to fully understand the situation, the lattice inputs for the form factor shape, not just the value in the zero-recoil limit, will play a crucial role. Several lattice projects are working toward this direction. At this conference, the status is reported by RBC/UKQCD (Witzel et al. [41]), JLQCD (Kaneko et al. [63]), HPQCD (McLean et al. [65]), Fermilab/MILC (Vaquero et al. [66], see also [67]), as well as the Seoul group (Park et al. [68]). 


\section{More challenges}

\subsection{Short-distance physics}

So far, most lattice calculations have focused on the properties of ground-state hadrons, such as their masses or form factors. In the real world, there are far more states of excited hadrons or scattering hadrons. In the perturbative analysis of QCD, one considers high-energy or shortdistance quark (and gluon) interactions and relates them to the experimental data after summing over all possible hadronic final states. The best-known example is the $R$-ratio, i.e. the ratio of the $e^{+} e^{-} \rightarrow q \bar{q}$ cross section to $e^{+} e^{-} \rightarrow \mu^{+} \mu^{-}$. Using the analyticity of the vacuum polarization function $\Pi\left(q^{2}\right)$, defined through $\left(q_{\mu} q_{v}-q^{2} g_{\mu v}\right) \Pi\left(q^{2}\right)=i \int d^{4} x e^{i q x}\left\langle 0\left|T\left[j_{\mu}(x) j_{v}(0)\right]\right| 0\right\rangle$, one can write down the equality

$$
\left.\frac{1}{n !}\left(\frac{\partial}{\partial q^{2}}\right)^{n} \Pi\left(q^{2}\right)\right|_{q^{2}=0}=\frac{1}{\pi} \int_{s_{\min }}^{\infty} d s \frac{\operatorname{Im} \Pi(s)}{s^{n+1}} .
$$

Here, let us restrict ourselves to the $c \bar{c}$ final states. The right-hand side contains the imaginary part $\operatorname{Im} \Pi(s)$, which is related to the sum over all possible final states of $e^{+} e^{-} \rightarrow c \bar{c}$. The left-hand side is the vacuum polarization function at $q^{2}=0$, far below the singularities - poles and cuts. Since $\Pi\left(q^{2}=0\right)$ is a short-distance quantity characterized by the length scale of $1 / m_{c}$, one can use the perturbative expansion to evaluate. This is the principle of the quarkonium sum rules, a version of the QCD sum rule $[69,70]$ applied for quarkonium. The method has been used to determine the charm quark mass, for instance, using the experimental data for $e^{+} e^{-} \rightarrow c \bar{c}$ (see [71, 72] for the most recent works).

The same perturbative expansion of the charmonium vacuum polarization function may be compared with the lattice data. Namely, one can replace the experimental data by the lattice results obtained directly at $q^{2}=0$. The calculation is free from ultraviolet divergences when $n>1$. The derivatives in terms of $q^{2}$ in (4.1) are transformed to the temporal moments of the charmonium correlator on the lattice, $\sum_{t} t^{2 n} G(t)$, which is straightforward to construct from the lattice data of the correlator $G(t)$. Since $G(t)$ decays exponentially in Euclidean time $t$ by the charmonium mass, the sum is saturated by the short-distance region $t \sim n / m_{c}$.

The pioneering work of the HPQCD collaboration $[73,74,75]$ opened a new application of lattice QCD for short-distance quantities. Fundamental parameters in QCD, $\alpha_{s}(\mu)$ and $m_{c}(\mu)$, defined in the $\overline{\mathrm{MS}}$ scheme were extracted directly from the equality between the lattice data and perturbative expansion for $d^{n} \Pi\left(q^{2}\right) / d q^{2 n}$. (The same method has also been utilized more recently by $[76,77]$.)

It also implies that one can compare the lattice data obtained in the short-distance region with the experimental data, without recourse to the perturbative expansion. Such a test has been performed and an agreement between the lattice results and experimental data has been confirmed $[78,76]$. Thus, triangular links connecting three sectors, i.e. experimental data, perturbation theory and lattice calculation, are established for this quantity, which is yet another evidence of the success of QCD.

The study of short-distance quantities necessarily involves a sum over hadronic final states. In this sense, the relevant process is inclusive. Namely, one does not specify any particular final state but treats all possible final states with a given quantum number added with some weight over 
a momentum variable. Such analysis is not limited to the $R$-ratio (or equivalently the vacuum polarization function), and here I would like to discuss an application to inclusive semi-leptonic decays.

In order to be specific, let us consider the inclusive decay process $B \rightarrow X_{c} \ell v$ with $X_{c}$ representing any possible state with a charm quark, such as $D^{(*)}, D \pi, D \pi \pi$, and so on. This process is more complicated than $e^{+} e^{-} \rightarrow c \bar{c}$, as there are two independent kinematical variables $q^{2}$ and $p_{B} \cdot q$. Here, $q^{\mu}$ is a momentum transfer to the lepton pair $\ell v$ and $p_{B}^{\mu}$ denotes the momentum of the initial $B$ meson.

The partial decay rate is proportional to $\left|V_{c b}\right|^{2}$ as well as to the structure function

$$
W_{\mu v}=\sum_{X_{c}}(2 \pi)^{3} \delta^{(4)}\left(p_{B}-q-p_{X}\right) \frac{1}{2 M_{B}}\left\langle B\left(p_{B}\right)\left|J_{\mu}^{\dagger}(0)\right| X_{c}\right\rangle\left\langle X_{c}\left|J_{v}(0)\right| B\left(p_{B}\right)\right\rangle,
$$

where the sum is over all possible final states $X_{c}$ with momentum specified by $p_{X}=p_{B}-q$ and $J_{\mu}$ is the weak current $\bar{c} \gamma_{\mu}\left(1-\gamma_{5}\right) b$. Using the optical theorem $-\frac{1}{\pi} \operatorname{Im} T=W$, one can relate the structure function (4.2) to the forward scattering matrix element

$$
T_{\mu \nu}=i \int d^{4} x e^{-i q x} \frac{1}{2 M_{B}}\left\langle B\left(p_{B}\right)\left|T\left\{J_{\mu}^{\dagger}(x) J_{v}(0)\right\}\right| B\left(p_{B}\right)\right\rangle .
$$

An important question is, then, whether this matrix element is calculable on the lattice.

In the region of $q^{2}$ and $p_{B} \cdot q$ where the physical processes occur, the matrix element $T_{\mu \nu}$ develops an imaginary part, which is not easily accessible on the Euclidean lattice. Instead, we consider the region of $p_{B} \cdot q$ for which the energy injected to the final $X_{c}$ state is not sufficient to produce real states. In other words, for the lattice calculation we take the region $v \cdot\left(p_{B}-\right.$ $q)<M_{D}$. (Here, $v^{\mu}=p_{B}^{\mu} / M_{B}$ denotes the four-velocity of the initial $B$ meson, so that the inner product $v \cdot\left(p_{B}-q\right)$ represents the energy given to $X_{c}$.) The matrix element in this unphysical kinematical region may be related to the physical decay amplitude, i.e. the imaginary part of $T_{\mu \nu}$, using Cauchy's integral of the form

$$
T(v \cdot q)=\int_{-\infty}^{(v \cdot q)_{\max }} \frac{d\left(v \cdot q^{\prime}\right)}{\pi} \frac{\operatorname{Im} T\left(v \cdot q^{\prime}\right)}{v \cdot q^{\prime}-v \cdot q}
$$

where I explicitly write the dependence on $v \cdot q$ while assuming fixed $q^{2}$.

Lattice calculation of the relevant matrix element is straightforward though more costly than the standard form factor calculations. One needs to calculate four-point functions to obtain

$$
C_{\mu v}^{J J}(t ; \mathbf{q})=\int d^{3} \mathbf{x} e^{i \mathbf{q} \cdot \mathbf{x}} \frac{1}{2 M_{B}}\left\langle B(\mathbf{0})\left|J_{\mu}^{\dagger}(\mathbf{x}, t) J_{v}(0)\right| B(\mathbf{0})\right\rangle .
$$

It is a function of a time-separation between the two inserted currents $J_{\mu}^{\dagger}$ and $J_{v}$. Then, the matrix element at the unphysical kinematical point may be constructed using a "Fourier" (or Laplace) transform

$$
T_{\mu v}^{J J}(\omega, \mathbf{q})=\int_{0}^{\infty} d t e^{\omega t} C_{\mu \nu}^{J J}(t ; \mathbf{q}) .
$$

Then, it may be compared with the physical amplitude through the relation (4.4). This is the strategy proposed in [79]. (The same strategy may also be applied to the study of (not-so) deep inelastic scattering. See also [80].) 


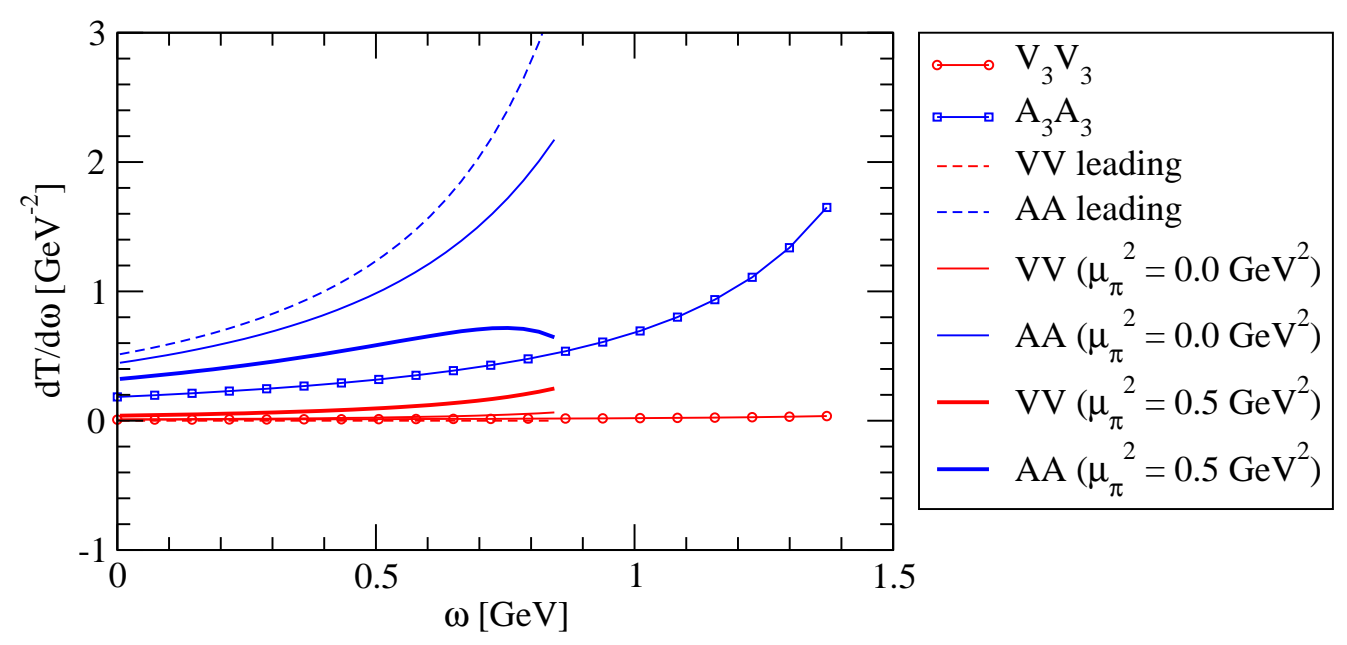

Figure 4: Forward-scattering matrix elements $d T_{k k} / d \omega$ as a function of the energy $\omega$ injected to the final charmed state. Lattice data are plotted for vector $V_{k}$ (red circles) and axial-vector $A_{k}$ (blue squares) channels. Spatial recoil momentum is set to zero. Curves are expectations from heavy quark expansion. See the text for details.

Results for $d T_{k k} / d \omega$ are plotted in Figure 4. Lattice data for the spatial vector (red circles) and axial-vector (blue squares) channels are plotted together with the expectation from the heavy quark expansion to $O\left(1 / \mathrm{m}^{2}\right)$ [81, 82]. The $b$ quark mass is lower than its physical value, $m_{b} \sim 2.4 m_{c}$, and we set the spatial recoil momentum $\mathbf{q}$ to zero. The axial-vector channel shows a significant contribution from the $D^{*}$ intermediate state. Indeed, it develops a pole $\sim 1 /\left(\omega-m_{D^{*}}\right)^{2}$ towards larger $\omega$. The vector channel, on the hand, is nearly vanishing. (Precisely speaking, the vector channel is small but non-zero representing the excited-state $D^{* *}$ contribution.)

The lattice results are compared with the expectation from the heavy quark expansion [81, 82] (curves in Figure 4). The dashed curves are those of the leading order, while the solid curves includes the corrections of $O\left(1 / m_{b}^{2}\right)$. At this order, two parameters characterizing the $B$ meson bound state appear, i.e. $\mu_{\pi}^{2}=\frac{1}{2 M_{B}}\left\langle B\left|\bar{b}(i \vec{D})^{2} b\right| B\right\rangle$ and $\mu_{G}^{2}=\frac{1}{2 M_{B}}\left\langle B\left|\bar{b}_{2} \frac{i}{2} \sigma_{\mu \nu} G^{\mu v} b\right| B\right\rangle$. We set $\mu_{G}^{2}$ to the value determined from the $B-B^{*}$ mass splitting, while $\mu_{\pi}^{2}$ is more uncertain and we took two nominal values $0 \mathrm{GeV}^{2}$ (thin lines) and $0.5 \mathrm{GeV}^{2}$ (thick lines). As can be seen clearly in the plot, the leading order estimate is far apart from the lattice data, and there is a trend that the $1 / \mathrm{m}^{2}$ correction makes them closer. It would be interesting to see how the next order works, and, more importantly, the perturbative corrections are to be included to have more realistic comparison. The work in that direction is underway [83].

There is another proposal for the lattice study of inclusive decay. It utilizes the lattice correlator $C_{\mu \nu}^{J J}(t ; \mathbf{q})$ in a different way. If one can solve the inverse problem

$$
C(t)=\int_{0}^{\infty} d \omega e^{-\omega t}\left(-\frac{1}{\pi} \operatorname{Im} T(\omega)\right)
$$

the physical amplitude $\operatorname{Im} T(\omega)$ may be extracted from the Euclidean lattice data $C(t)$. In practice, 
this is not possible without having infinitely dense data available for $C(t)$. Instead, one may try to extract $T(\omega)$ smeared over some small interval. A systematic way of doing this was proposed by [84].

\subsection{Long-distance effects}

Another interesting area where lattice calculation may give a significant impact is a study of long-distance effects. A well-known example is the $B \rightarrow K \ell^{+} \ell^{-}$decay, which may have a significant contribution from intermediate $b \rightarrow s \bar{c} c$ state due to resonances $J / \psi, \psi^{\prime}$, etc. Since they can occur through a Cabbibo-allowed operator $\mathscr{O}_{2}=\left(\bar{s} \gamma_{\mu} P_{L} c\right)\left(\bar{c} \gamma^{\mu} P_{L} b\right)$, the corresponding amplitude is large especially near the resonances. In the experimental analysis one therefore eliminate the regions of $q^{2}$, invariant mass squared of the final-state leptons, close to the corresponding resonances. It is not entirely clear, however, how much effects are left outside of these regions, and lattice calculation may shed new light on this problem. (For a review of the situation, see, for instance [85]. It also emphasizes that the factorization approximation doesn't reproduce the available experimental data.)

Lattice study of the long-distance effects of this type has been initiated by the RBC/UKQCD collaboration [86, 87] for $K \rightarrow \pi \ell^{+} \ell^{-}$. The problem is simpler for $B \rightarrow K \ell^{+} \ell^{-}$as long as the effect of the $c \bar{c}$ contributions is concerned. Essentially, one needs to calculate the matrix element of the form

$$
\int_{0}^{\infty} d t e^{\omega t} \int d^{3} \mathbf{x} e^{-i \mathbf{q} \cdot \mathbf{x}}\left\langle K(\mathbf{q})\left|J_{\mu}^{(\mathrm{em})}(t, \mathbf{x}) \mathscr{H}(0)\right| B(\mathbf{0})\right\rangle,
$$

where the weak effective Hamiltonian $\mathscr{H}$ relevant to the process of interest contains the fourfermion operator $\left(\bar{c} \gamma_{\mu} P_{L} b\right)\left(\bar{s} \gamma_{\mu} P_{L} c\right)$ as well as $\left(\bar{c} \gamma_{\mu} P_{L} c\right)\left(\bar{s} \gamma_{\mu} P_{L} b\right)$. The integral over $t$ with a factor $e^{\omega t}$ specifies the energy $\omega$ inserted to the electromagnetic current $J_{\mu}^{(\mathrm{em})}=\bar{c} \gamma_{\mu} c$. In order that the integral stays finite, the value of $\omega$ must be lower than the energy of $J / \psi$.

Before analyzing the amplitude (4.8), it is interesting to see how well the factorization assumption approximates the matrix element $\left\langle K(\mathbf{q})\left|J_{\mu}^{(\mathrm{em})}(x) \mathscr{H}(0)\right| B(\mathbf{0})\right\rangle$. Here, the factorization assumption implies that a matrix element of complex process approximated by a product of simpler matrix elements, e.g.

$$
\left\langle K(\mathbf{q})\left|J_{\mu}^{(\mathrm{em})}(x)\left(\bar{c} \gamma_{\mu} P_{L} c\right)\left(\bar{s} \gamma_{\mu} P_{L} b\right)(0)\right| B(\mathbf{0})\right\rangle \simeq\left\langle K(\mathbf{q})\left|\bar{s} \gamma_{\mu} P_{L} b(0)\right| B(\mathbf{0})\right\rangle\left\langle 0\left|J_{\mu}^{(\mathrm{em})}(x) \bar{c} \gamma_{\mu} P_{L} c(0)\right| 0\right\rangle .
$$

Here the $B \rightarrow K$ form factors and charmonium decay constant are used to express the more complicated process of $b \rightarrow s c \bar{c} \rightarrow s \ell^{+} \ell^{-}$. It may introduce an uncontrollable systematic error, as it ignores for instance the effect of rescattering of the final state $K$ with the charmonium.

By a lattice calculation, it turned out that the factorization is well satisfied for the operator $\mathscr{O}_{1}=$ $\left(\bar{c} \gamma_{\mu} P_{L} c\right)\left(\bar{s} \gamma_{\mu} P_{L} b\right)$, while an $O(1)$ violation is observed for $\mathscr{O}_{2}=\left(\bar{c} \gamma_{\mu} P_{L} b\right)\left(\bar{s} \gamma_{\mu} P_{L} c\right)$. Namely, a ratio of the matrix elements of $\mathscr{O}_{1}$ and $\mathscr{O}_{2}$ is expected to be $1 / 3$ in the factorization approximation, and a preliminary lattice data is more like zero [88]. A strong violation of the factorization approximation was found for the $\Delta I=3 / 2$ amplitude of $K \rightarrow \pi \pi$ and it may be a key for an understanding of the $\Delta I=1 / 2$ rule [89]. It would therefore be interesting to see how the subtle details of the strong interaction affects the amplitude of $B \rightarrow K \ell^{+} \ell^{-}$. 


\subsection{Non-local current insertions}

There are physical quantities that are related to a matrix element of bilocal operators. Both of the last two applications, the forward-scattering matrix element for inclusive decays and the nonlocal current insertions for $B \rightarrow K \ell \ell$, are examples of such quantities. There are more examples related to interesting physics applications.

One is a matrix element corresponding to the process $B \rightarrow \ell v \gamma$. This decay mode is special because by adding a photon one can avoid the helicity suppression of the leptonic decay $B \rightarrow \ell v$. It could therefore be significant even though one has to pay the penalty of $\alpha=1 / 137$. It may also provide an interesting testing ground for the lepton flavor universality. A lattice study of this decay mode has been presented at this conference [90].

Another such (and related) example is the calculation of the QED correction to the leptonic and semi-leptonic processes. The formulation to calculate the QED correction has been developed for pion decay constant $[91,92,93]$. Extension of the idea to the heavier mesons is discussed at this conference [94].

\section{Conclusions}

Lattice calculation for heavy flavor physics reached the stage of enabling precise calculations at a percent level. This is an integral part of the program to search for any limitations of the Standard Model. Experiments, such as LHCb and Belle II, are going to produce a lot of precise data in the coming years, and the lattice calculation has to follow by improving its precision to maximize the power of the new physics search.

The role of lattice calculation is not limited to improving the precision. There are many physical processes which would be more useful once the hadronic uncertainty is made under control. The long-distance effect to $B \rightarrow K \ell^{+} \ell^{-}$is a good example. By extending its application, lattice calculation would play the role to expand the horizon of flavor physics.

\section{Acknowledgments}

I thank C. Bouchard, B. Colquhoun, C. Davies, A. El-Khadra, P. Gambino, A. Juttner, T. Kaneko, J. Komijani, Z. Ligeti, K. Nakayama, C. Sachrajda, A. Soni, A. Vaquero for their inputs and discussions. This work is supported in part by JSPS KAKENHI Grant Number $18 \mathrm{H} 03710$.

\section{References}

[1] HFLAV Collaboration, Y. Amhis et. al., Averages of b-hadron, c-hadron, and $\tau$-lepton properties as of summer 2016, Eur. Phys. J. C77 (2017), no. 12895 [1612 . 07233].

[2] Heavy Flavour Averaging Group, Semileptonic B decays subgroup Collaboration, C. Bozzi, M. Rotondo, V. Luth, J. Dingfelder, C. Schwanda and P. Urquijo, Average of $R(D)$ and $R\left(D^{*}\right)$ for Summer 2018, .

[3] MILC Collaboration, J. A. Bailey et. al., $B \rightarrow D \ell v$ form factors at nonzero recoil and $\left|V_{c b}\right|$ from 2+1-flavor lattice QCD, Phys. Rev. D92 (2015), no. 3034506 [1503. 0 7237]. 
[4] HPQCD Collaboration, H. Na, C. M. Bouchard, G. P. Lepage, C. Monahan and J. Shigemitsu, $B \rightarrow$ Dlv form factors at nonzero recoil and extraction of $\left|V_{c b}\right|$, Phys. Rev. D92 (2015), no. 5054510 [1505.03925]. [Erratum: Phys. Rev.D93,no.11,119906(2016)].

[5] LHCb Collaboration, R. Aaij et. al., Test of lepton universality using $B^{+} \rightarrow K^{+} \ell^{+} \ell^{-}$decays, Phys. Rev. Lett. 113 (2014) 151601 [1406.6482].

[6] LHCb Collaboration, R. Aaij et. al., Test of lepton universality with $B^{0} \rightarrow K^{* 0} \ell^{+} \ell^{-}$decays, JHEP $\mathbf{0 8}$ (2017) 055 [1705.05802].

[7] LHCb Collaboration, R. Aaij et. al., Angular analysis of the $B^{0} \rightarrow K^{* 0} \mu^{+} \mu^{-}$decay using $3 \mathrm{fb}^{-1}$ of integrated luminosity, JHEP 02 (2016) 104 [1512. 044 42].

[8] Belle Collaboration, S. Wehle et. al., Lepton-Flavor-Dependent Angular Analysis of $B \rightarrow K^{*} \ell^{+} \ell^{-}$, Phys. Rev. Lett. 118 (2017), no. 11111801 [1612.05014].

[9] CMS Collaboration, A. M. Sirunyan et. al., Measurement of angular parameters from the decay $\mathrm{B}^{0} \rightarrow \mathrm{K}^{* 0} \mu^{+} \mu^{-}$in proton-proton collisions at $\sqrt{s}=8 \mathrm{TeV}$, Phys. Lett. $\mathrm{B} 781$ (2018) 517-541 [1710.02846].

[10] ATLAS Collaboration, M. Aaboud et. al., Angular analysis of $B_{d}^{0} \rightarrow K^{*} \mu^{+} \mu^{-}$decays in pp collisions at $\sqrt{s}=8 \mathrm{TeV}$ with the ATLAS detector, 1805.04000 .

[11] D. Du, A. X. El-Khadra, S. Gottlieb, A. S. Kronfeld, J. Laiho, E. Lunghi, R. S. Van de Water and R. Zhou, Phenomenology of semileptonic B-meson decays with form factors from lattice QCD, Phys. Rev. D93 (2016), no. 3034005 [1510.02349].

[12] S. Aoki et. al., Review of lattice results concerning low-energy particle physics, Eur. Phys. J. C77 (2017), no. 2112 [1607.00299].

[13] A. Bazavov et. al., B- and D-meson leptonic decay constants from four-flavor lattice QCD, Phys. Rev. D98 (2018), no. 7074512 [1712.09262].

[14] Fermilab Lattice, MILC Collaboration, A. Bazavov et. al., B-and D-meson decay constants from three-flavor lattice QCD, Phys. Rev. D85 (2012) 114506 [1112.3051].

[15] N. H. Christ, J. M. Flynn, T. Izubuchi, T. Kawanai, C. Lehner, A. Soni, R. S. Van de Water and O. Witzel, B-meson decay constants from 2+1-flavor lattice QCD with domain-wall light quarks and relativistic heavy quarks, Phys. Rev. D91 (2015), no. 5054502 [1 404.4670 ].

[16] Y. Aoki, T. Ishikawa, T. Izubuchi, C. Lehner and A. Soni, Neutral B meson mixings and B meson decay constants with static heavy and domain-wall light quarks, Phys. Rev. D91 (2015), no. 11 114505 [1406.6192].

[17] H. Na, C. J. Monahan, C. T. H. Davies, R. Horgan, G. P. Lepage and J. Shigemitsu, The B and $B_{S}$ Meson Decay Constants from Lattice QCD, Phys. Rev. D86 (2012) 034506 [1202 . 4914].

[18] HPQCD Collaboration, R. J. Dowdall, C. T. H. Davies, R. R. Horgan, C. J. Monahan and J. Shigemitsu, B-Meson Decay Constants from Improved Lattice Nonrelativistic QCD with Physical $u$, d, s, and c Quarks, Phys. Rev. Lett. 110 (2013), no. 22222003 [1302. 2644 ].

[19] HPQCD, UKQCD Collaboration, E. Follana, Q. Mason, C. Davies, K. Hornbostel, G. P. Lepage, J. Shigemitsu, H. Trottier and K. Wong, Highly improved staggered quarks on the lattice, with applications to charm physics, Phys. Rev. D75 (2007) 054502 [hep-lat/ 06100 92].

[20] A. X. El-Khadra, A. S. Kronfeld and P. B. Mackenzie, Massive fermions in lattice gauge theory, Phys. Rev. D55 (1997) 3933-3957 [hep-lat/9604004]. 
[21] C. McNeile, C. T. H. Davies, E. Follana, K. Hornbostel and G. P. Lepage, High-Precision $f_{B_{s}}$ and HQET from Relativistic Lattice QCD, Phys. Rev. D85 (2012) 031503 [1110 . 4510].

[22] G. P. Lepage, L. Magnea, C. Nakhleh, U. Magnea and K. Hornbostel, Improved nonrelativistic QCD for heavy quark physics, Phys. Rev. D46 (1992) 4052-4067 [hep-lat/920 5007 ].

[23] ALPHA Collaboration, J. Heitger and R. Sommer, Nonperturbative heavy quark effective theory, JHEP 02 (2004) 022 [hep-lat/ 0310035$].$

[24] B. Blossier, M. della Morte, N. Garron and R. Sommer, HQET at order 1/m: I. Non-perturbative parameters in the quenched approximation, JHEP 06 (2010) 002 [1 001 . 4 783].

[25] Alpha Collaboration, B. Blossier, M. Della Morte, N. Garron, G. von Hippel, T. Mendes, H. Simma and R. Sommer, HQET at order 1/m: II. Spectroscopy in the quenched approximation, JHEP $\mathbf{0 5}$ (2010) 074 [1004.2661].

[26] ALPHA Collaboration, B. Blossier, M. Della Morte, N. Garron, G. von Hippel, T. Mendes, H. Simma and R. Sommer, HQET at order 1/m: III. Decay constants in the quenched approximation, JHEP 12 (2010) 039 [1006.5816].

[27] G. P. Lepage, The Analysis of Algorithms for Lattice Field Theory, in Boulder ASI 1989:97-120, pp. 97-120, 1989.

[28] S. Hashimoto, Computation of the heavy - light decay constant using nonrelativistic lattice QCD, Phys. Rev. D50 (1994) 4639-4648 [hep-lat/9403028].

[29] R. Gupta, Y.-C. Jang, H.-W. Lin, B. Yoon and T. Bhattacharya, Axial Vector Form Factors of the Nucleon from Lattice QCD, Phys. Rev. D96 (2017), no. 11114503 [1705. 06834 ].

[30] O. Bar, Multi-hadron-state contamination in nucleon observables from chiral perturbation theory, EPJ Web Conf. 175 (2018) 01007 [1708.00380].

[31] O. Bär, Nucleon-pion-state contribution in lattice calculations of the nucleon charges $g_{A}, g_{T}$ and $g_{S}$, Phys. Rev. D94 (2016), no. 5054505 [1606.09385].

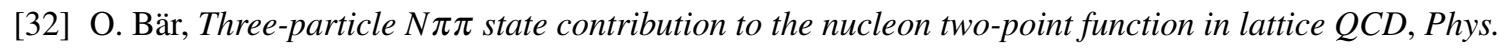
Rev. D97 (2018), no. 9094507 [1802. 10442 ].

[33] O. Bar, Nucleon-pion-state contamination in lattice calculations of the axial form factors of the nucleon, PoS LATTICE2018 (2018) 061 [1808 . 08738].

[34] W. Detmold, C. Lehner and S. Meinel, $\Lambda_{b} \rightarrow p \ell^{-} \bar{v}_{\ell}$ and $\Lambda_{b} \rightarrow \Lambda_{c} \ell^{-} \bar{v}_{\ell}$ form factors from lattice QCD with relativistic heavy quarks, Phys. Rev. D92 (2015), no. 3034503 [1503.01421].

[35] J. M. Flynn, T. Izubuchi, T. Kawanai, C. Lehner, A. Soni, R. S. Van de Water and O. Witzel, $B \rightarrow \pi \ell v$ and $B_{s} \rightarrow K \ell v$ form factors and $\left|V_{u b}\right|$ from 2+1-flavor lattice $Q C D$ with domain-wall light quarks and relativistic heavy quarks, Phys. Rev. D91 (2015), no. 7074510 [1501.05373].

[36] Fermilab Lattice, MILC Collaboration, J. A. Bailey et. al., $\left|V_{u b}\right|$ from $B \rightarrow \pi \ell v$ decays and (2+1)-flavor lattice QCD, Phys. Rev. D92 (2015), no. 1014024 [1503.07839].

[37] F. Bahr, D. Banerjee, M. Koren, H. Simma and R. Sommer, Extraction of the bare form factors for the semi-leptonic $B_{s}$ decays, PoS LATTICE2016 (2016) 295 [1 701 . 032 99].

[38] Z. Gelzer, Semileptonic decays of $B_{s}$ mesons to light pseudoscalar mesons on four-flavor HISQ ensembles, PoS LATTICE2018 (2018) 289.

[39] Z. Gelzer et. al., Semileptonic B-meson decays to light pseudoscalar mesons on the HISQ ensembles, EPJ Web Conf. 175 (2018) 13024 [1710.09442]. 
[40] Fermilab Lattice, MILC Collaboration, Y. Liu et. al., $B_{s} \rightarrow K \ell v$ Form Factors with 2+1 Flavors, EPJ Web Conf. 175 (2018) 13008 [1711.08085].

[41] O. Witzel, Semi-leptonic form factors for $B_{s} \rightarrow K \ell v$ and $B_{S} \rightarrow D_{s} \ell v$, PoS LATTICE2018 (2018) 290.

[42] B. Colquhoun, S. Hashimoto and T. Kaneko, Heavy quark scaling of $B \rightarrow \pi \ell v$ form factors with Möbius domain wall fermions, PoS LATTICE2018 (2018) 274 [1811. 00227$].$

[43] B. Colquhoun, S. Hashimoto and T. Kaneko, $B \rightarrow \pi \ell v$ with Möbius Domain Wall Fermions, EPJ Web Conf. 175 (2018) 13004 [1710.07094].

[44] C. Bouchard, $B \rightarrow \pi \ell v$ and $B \rightarrow \pi \ell \ell$ decays with HISQ/NRQCD valence quarks on $N_{f}=2+1$ asqtad ensembles, PoS LATTICE2018 (2018) 288.

[45] C. J. Monahan, C. M. Bouchard, G. P. Lepage, H. Na and J. Shigemitsu, Form factor ratios for $B_{s} \rightarrow K \ell v$ and $B_{s} \rightarrow D_{s} \ell v$ semileptonic decays and $\left|V_{u b} / V_{c b}\right|, 1808.09285$.

[46] V. Lubicz, L. Riggio, G. Salerno and S. Simula, Hypercubic effects in semileptonic decays of heavy mesons, toward $B \rightarrow \pi \ell v$, with $N_{f}=2+1+1$ Twisted fermions, PoS LATTICE2018 (2018) 287 [1811.10268].

[47] ETM Collaboration, V. Lubicz, L. Riggio, G. Salerno, S. Simula and C. Tarantino, Tensor form factor of $D \rightarrow \pi(K) \ell v$ and $D \rightarrow \pi(K) \ell \ell$ decays with $N_{f}=2+1+1$ twisted-mass fermions, Phys. Rev. D98 (2018), no. 1014516 [1803.04807].

[48] N. Isgur and M. B. Wise, Weak Decays of Heavy Mesons in the Static Quark Approximation, Phys. Lett. B232 (1989) 113-117.

[49] N. Isgur and M. B. Wise, WEAK TRANSITION FORM-FACTORS BETWEEN HEAVY MESONS, Phys. Lett. B237 (1990) 527-530.

[50] M. E. Luke, Effects of subleading operators in the heavy quark effective theory, Phys. Lett. $\mathbf{B 2 5 2}$ (1990) 447-455.

[51] S. Hashimoto, A. X. El-Khadra, A. S. Kronfeld, P. B. Mackenzie, S. M. Ryan and J. N. Simone, Lattice QCD calculation of $\bar{B} \rightarrow D \ell \bar{v}$ decay form-factors at zero recoil, Phys. Rev. D61 (1999) 014502 [hep-ph/9906376].

[52] S. Hashimoto, A. S. Kronfeld, P. B. Mackenzie, S. M. Ryan and J. N. Simone, Lattice calculation of the zero recoil form-factor of $\bar{B} \rightarrow D^{*} \ell v$ : Toward a model independent determination of $\left|V_{c b}\right|$, Phys. Rev. D66 (2002) 014503 [hep-ph/ 0110253$].$

[53] Fermilab Lattice, MILC Collaboration, J. A. Bailey et. al., Update of $\left|V_{c b}\right|$ from the $\bar{B} \rightarrow D^{*} \ell \bar{v}$ form factor at zero recoil with three-flavor lattice QCD, Phys. Rev. D89 (2014), no. 11114504 [1403.0635].

[54] HPQCD Collaboration, J. Harrison, C. Davies and M. Wingate, Lattice QCD calculation of the $B_{(s)} \rightarrow D_{(s)}^{*} \ell v$ form factors at zero recoil and implications for $\left|V_{c b}\right|$, Phys. Rev. D97 (2018), no. 5 $054502[1711.11013]$.

[55] Belle Collaboration, A. Abdesselam et. al., Precise determination of the CKM matrix element $\left|V_{c b}\right|$ with $\bar{B}^{0} \rightarrow D^{*+} \ell^{-} \bar{v}_{\ell}$ decays with hadronic tagging at Belle, 1702.01521.

[56] D. Bigi, P. Gambino and S. Schacht, A fresh look at the determination of $\left|V_{c b}\right|$ from $B \rightarrow D^{*} \ell v$, Phys. Lett. B769 (2017) 441-445 [1703. 06124$].$

[57] B. Grinstein and A. Kobach, Model-Independent Extraction of $\left|V_{c b}\right|$ from $\bar{B} \rightarrow D^{*} \ell \bar{v}$, Phys. Lett. B771 (2017) 359-364 [1703.08170]. 
[58] F. U. Bernlochner, Z. Ligeti, M. Papucci and D. J. Robinson, Tensions and correlations in $\left|V_{c b}\right|$ determinations, Phys. Rev. D96 (2017), no. 9091503 [1708.07134].

[59] I. Caprini, L. Lellouch and M. Neubert, Dispersive bounds on the shape of $\bar{B} \rightarrow D^{(*)} \ell \bar{v}$ form-factors, Nucl. Phys. B530 (1998) 153-181 [hep-ph/9712417].

[60] C. G. Boyd, B. Grinstein and R. F. Lebed, Model independent extraction of $\left|V_{c b}\right|$ using dispersion relations, Phys. Lett. B353 (1995) 306-312 [hep-ph/ 9504235$].$

[61] S. Okubo and I.-F. Shih, Exact inequality and test of chiral sw(3) theory in k-l-3 decay problem, Phys. Rev. D4 (1971) 2020-2029.

[62] C. Bourrely, B. Machet and E. de Rafael, Semileptonic Decays of Pseudoscalar Particles $\left(M \rightarrow M^{\prime} \ell v_{\ell}\right)$ and Short Distance Behavior of Quantum Chromodynamics, Nucl. Phys. B189 (1981) 157-181.

[63] JLQCD Collaboration, T. Kaneko, Y. Aoki, B. Colquhoun, H. Fukaya and S. Hashimoto, $B \rightarrow D^{(*)} \ell v$ form factors from $N_{f}=2+1$ QCD with Möbius domain-wall quarks, PoS LATTICE2018 (2018) 311 [1811.00794].

[64] D. Bigi, P. Gambino and S. Schacht, $R\left(D^{*}\right),\left|V_{c b}\right|$, and the Heavy Quark Symmetry relations between form factors, JHEP 11 (2017) 061 [1707.09509].

[65] E. McLean, $B_{S} \rightarrow D_{s} \ell v$ form factors using heavy HISQ quarks, PoS LATTICE2018 (2018) 281.

[66] A. Vaquero, $B \rightarrow D^{*} \ell v$ at non-zero recoil, PoS LATTICE2018 (2018) 282.

[67] A. Vaquero Avilés-Casco, C. DeTar, D. Du, A. El-Khadra, A. S. Kronfeld, J. Laiho and R. S. Van de Water, $\bar{B} \rightarrow D^{*} \ell \bar{v}$ at Non-Zero Recoil, EPJ Web Conf. 175 (2018) 13003 [1710. 09817 ].

[68] T. Bhattacharya, R. Gupta, S. Park, Y. Jang, J. Bailey, B. Choi, H. Jeong, S. Jwa, S. Lee, W. Lee, $\mathrm{J}$. Pak and J. Leem, Update on $B \rightarrow D^{*} \ell v$ form factor at zero-recoil using the Oktay-Kronfeld action, PoS LATTICE2018 (2018) 283.

[69] M. A. Shifman, A. I. Vainshtein and V. I. Zakharov, QCD and Resonance Physics. Theoretical Foundations, Nucl. Phys. B147 (1979) 385-447.

[70] M. A. Shifman, A. I. Vainshtein and V. I. Zakharov, QCD and Resonance Physics: Applications, Nucl. Phys. B147 (1979) 448-518.

[71] K. G. Chetyrkin, J. H. Kuhn, A. Maier, P. Maierhofer, P. Marquard, M. Steinhauser and C. Sturm, Charm and Bottom Quark Masses: An Update, Phys. Rev. D80 (2009) 074010 [0 907.2110 ].

[72] B. Dehnadi, A. H. Hoang, V. Mateu and S. M. Zebarjad, Charm Mass Determination from QCD Charmonium Sum Rules at Order $\alpha_{s}^{3}$, JHEP 09 (2013) 103 [1102.2264].

[73] HPQCD Collaboration, I. Allison et. al., High-Precision Charm-Quark Mass from Current-Current Correlators in Lattice and Continuum QCD, Phys. Rev. D78 (2008) 054513 [0 805.2999 ].

[74] C. McNeile, C. T. H. Davies, E. Follana, K. Hornbostel and G. P. Lepage, High-Precision $c$ and b Masses, and QCD Coupling from Current-Current Correlators in Lattice and Continuum QCD, Phys. Rev. D82 (2010) 034512 [1004 . 4285].

[75] B. Chakraborty, C. T. H. Davies, B. Galloway, P. Knecht, J. Koponen, G. C. Donald, R. J. Dowdall, G. P. Lepage and C. McNeile, High-precision quark masses and QCD coupling from $n_{f}=4$ lattice QCD, Phys. Rev. D91 (2015), no. 5054508 [1408.4169]. 
[76] K. Nakayama, B. Fahy and S. Hashimoto, Short-distance charmonium correlator on the lattice with Möbius domain-wall fermion and a determination of charm quark mass, Phys. Rev. D94 (2016), no. 5 054507 [1606.01002].

[77] Y. Maezawa and P. Petreczky, Quark masses and strong coupling constant in 2+1 flavor QCD, Phys. Rev. D94 (2016), no. 3034507 [1606.08798].

[78] G. C. Donald, C. T. H. Davies, R. J. Dowdall, E. Follana, K. Hornbostel, J. Koponen, G. P. Lepage and C. McNeile, Precision tests of the J/ $\psi$ from full lattice QCD: mass, leptonic width and radiative decay rate to $\eta_{c}$, Phys. Rev. D86 (2012) 094501 [1208.2855].

[79] S. Hashimoto, Inclusive semi-leptonic B meson decay structure functions from lattice QCD, PTEP 2017 (2017), no. 5 053B03 [1703.01881].

[80] A. J. Chambers, R. Horsley, Y. Nakamura, H. Perlt, P. E. L. Rakow, G. Schierholz, A. Schiller, K. Somfleth, R. D. Young and J. M. Zanotti, Nucleon Structure Functions from Operator Product Expansion on the Lattice, Phys. Rev. Lett. 118 (2017), no. 24242001 [1703 . 01153 ].

[81] A. V. Manohar and M. B. Wise, Inclusive semileptonic B and polarized Lambda(b) decays from QCD, Phys. Rev. D49 (1994) 1310-1329 [hep-ph/9308246].

[82] B. Blok, L. Koyrakh, M. A. Shifman and A. I. Vainshtein, Differential distributions in semileptonic decays of the heavy flavors in QCD, Phys. Rev. D49 (1994) 3356 [hep-ph/ 930724 7]. [Erratum: Phys. Rev.D50,3572(1994)].

[83] B. Colquhoun, P. Gambino, S. Hashimoto and T. Kaneko, Inclusive decay structure function for $B \rightarrow X_{c} \ell v:$ a comparison of a lattice calculation with the heavy quark expansion, PoS LATTICE2018 (2018) 307.

[84] M. T. Hansen, H. B. Meyer and D. Robaina, From deep inelastic scattering to heavy-flavor semileptonic decays: Total rates into multihadron final states from lattice QCD, Phys. Rev. D96 (2017), no. 9094513 [1704.08993].

[85] J. Lyon and R. Zwicky, Resonances gone topsy turvy - the charm of QCD or new physics in $b \rightarrow s \ell^{+} \ell^{-} ?, 1406.0566$.

[86] RBC, UKQCD Collaboration, N. H. Christ, X. Feng, A. Portelli and C. T. Sachrajda, Prospects for a lattice computation of rare kaon decay amplitudes: $K \rightarrow \pi \ell^{+} \ell^{-}$decays, Phys. Rev. D92 (2015), no. 9 094512 [1507.03094].

[87] N. H. Christ, X. Feng, A. Juttner, A. Lawson, A. Portelli and C. T. Sachrajda, First exploratory calculation of the long-distance contributions to the rare kaon decays $K \rightarrow \pi \ell^{+} \ell^{-}$, Phys. Rev. D94 (2016), no. 11114516 [1608.07585].

[88] K. Nakayama and S. Hashimoto, Test of factorization for the long-distance effects from charmonium in $B \rightarrow K \ell \ell, P o S$ LATTICE2018 (2018) 221.

[89] RBC, UKQCD Collaboration, P. A. Boyle et. al., Emerging understanding of the $\Delta I=1 / 2$ Rule from Lattice QCD, Phys. Rev. Lett. 110 (2013), no. 15152001 [1212.1474].

[90] A. Soni, Flavor anomalies \& the lattice, PoS LATTICE2018 (2018) 292.

[91] N. Carrasco, V. Lubicz, G. Martinelli, C. T. Sachrajda, N. Tantalo, C. Tarantino and M. Testa, QED Corrections to Hadronic Processes in Lattice QCD, Phys. Rev. D91 (2015), no. 7074506 [1502.00257]. 
[92] V. Lubicz, G. Martinelli, C. T. Sachrajda, F. Sanfilippo, S. Simula and N. Tantalo, Finite-Volume QED Corrections to Decay Amplitudes in Lattice QCD, Phys. Rev. D95 (2017), no. 3034504 [1611.08497].

[93] D. Giusti, V. Lubicz, G. Martinelli, C. T. Sachrajda, F. Sanfilippo, S. Simula, N. Tantalo and C. Tarantino, First lattice calculation of the QED corrections to leptonic decay rates, Phys. Rev. Lett. 120 (2018), no. 7072001 [1711.06537].

[94] D. Giusti, V. Lubicz, G. Martinelli, C. Sachrajda, F. Sanfilippo, S. Simula and N. Tantalo, Radiative corrections to decay amplitudes in lattice QCD, PoS LATTICE2018 (2018) 266 [1811. 06364 ]. 